C. Mazier, University of Texas

M. Rashed, X PA

U. Ravaioli, University of Illinois

C. Snell, $X$ TM

L. Thode, $X$

Submitted to:

DOE Office of Scientific and Technical Information (OSTI)

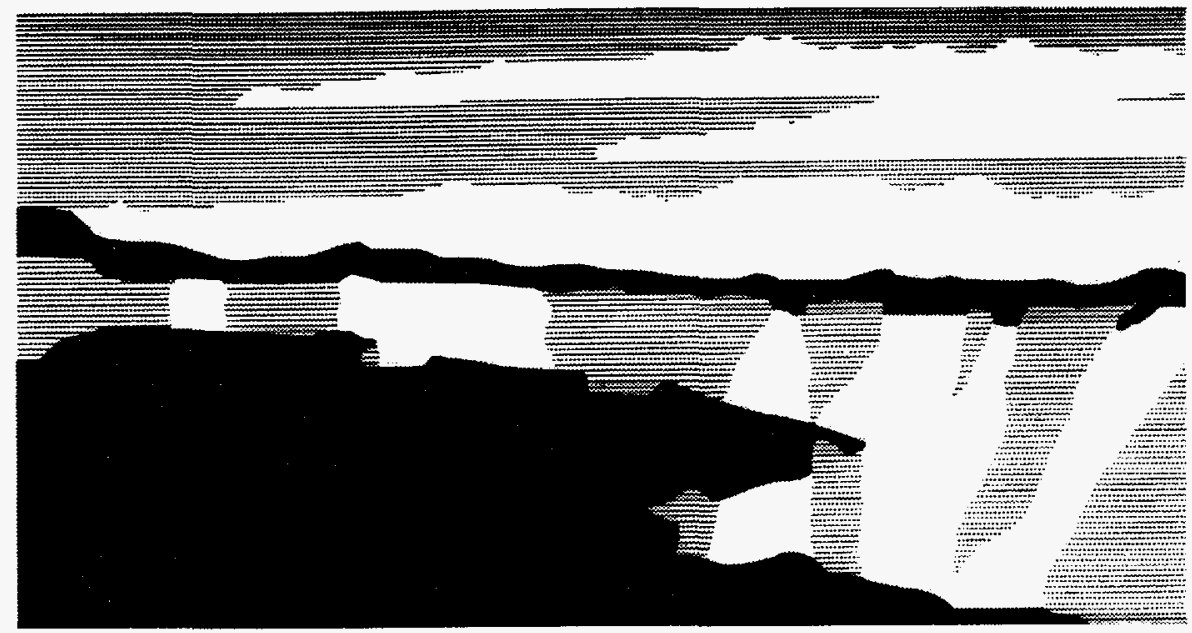

Los Alamos National Laboratory, an affirmative action/equal opportunity employer, is operated by the University of California for the U.S. Department of Energy under contract W-7405-ENG-36. By acceptance of this article, the publisher recognizes that the U.S. Government retains a nonexclusive, royaltyfree license to publish or reproduce the published form of this contribution, or to allow others to do so, for U.S. Government purposes. The Los Alamos National Laboratory requests that the publisher identify this article as work performed under the angign of 


\section{DISCLAIMER}

Portions of this document may be illegible in electronic image products. Images are produced from the best available original document. 


\section{DISCLAIMER}

This report was prepared as an account of work sponsored by an agency of the United States Government. Neither the United States Government nor any agency thereof, nor any of their employees, makes any warranty, express or implied, or assumes any legal liability or responsibility for the accuracy, completeness, or usefulness of any information, apparatus, product, or process disclosed, or represents that its use would not infringe privately owned rights. Reference herein to any specific commercial product, process, or service by trade name, trademark, manufacturer, or otherwise does not necessarily constitute or imply its endorsement, recommendation, or favoring by the United States Government or any agency thereof. The views and opinions of authors expressed herein do not necessarily state or reflect those of the United States Government or any agency thereof. 


\title{
Investigation of Charge Transport and Electromagnetic Effects in Advanced Microelectronics and Optoelectronics
}

\author{
T. Kwan*, T. Booth, M. Gray, K. Hess (University of Illinois), \\ C. Mazier (University of Texas), M. Rashed, U. Ravaioli (University of Illinois), \\ C. Snell, and L. Thode
}

\begin{abstract}
This is the final report of a three-year, Laboratory-Directed Research and Development (LDRD) project at the Los Alamos National Laboratory (LANL). The next generation of electronic microchips will utilize components with submicron feature size and optoelectronic devices with picosecond response time. Fundamental understanding of the device performance can only be obtained through first principles physics modeling of charge transport and electromagnetic effects in realistic geometries with material interfaces and dispersive properties. We have developed a general model incorporating important physics such as charge transport processes in materials with multilevel band structures and electromagnetic effects to simulate device characteristics. Accurate treatment of material interfaces and boundaries is included. The Monte Carlo charge transport is coupled self-consistently to Maxwell's equations to accurately model scattering processes in the presence of an externally biased potential. This detailed multidimensional simulation capability is compared with and verified by experimental data, and could become an industrial standard for benchmarking and improving the "reduced model" codes used for semiconductor design. Specific tasks are the extension of existing capabilities in particle-in-cell plasma simulation technique and Monte Carlo charge transport to study the physics of charged particle dynamics in realistic microelectronic devices, such as bipolar semiconductors, heterojunction transistors, and optoelectronic switches. Our approach has been based on the coupled particle-in-cell/Monte Carlo technique, which can simultaneously treat both electromagnetic wave propagation and charged-particle transport.
\end{abstract}

*Principal investigator, e-mail: tjtk@lanl.gov 


\section{Background and Research Objectives}

The standard method for modeling and design of microelectronics involves the solution of a coupled set of differential equations representing the behavior of individual elements in the circuit. The electrical parameters adopted for each element may be constant, or may vary with frequency. The division of the circuit into separate elements and the parameters assumed are often somewhat arbitrary. Moreover, this approach does not take account of the underlying physics that actually determines the behavior of semiconductor components.

A more sophisticated approach utilizes the finite-difference fluid continuity equations for current flow within the device, coupled to a solution of Poisson's equation for the electric field. This method does take proper account of the distributed nature and complex geometry of actual devices. Physics of the charge transport in the material enters through the equivalent fluid parameters of charge mobility and diffusivity, which are determined heuristically. Perhaps the most serious weakness of the fluid approach arises from the assumption that the charge carriers are massless and respond instantaneously to changes in the electric field. It is now well known that finite scattering times and velocity overshoot effects are crucial in the behavior of fast components. The time required for evolution of the carrier distribution and establishment of steady state mobility behavior is 1-5 picoseconds, corresponding to transport distances of a fraction of a micron. These are precisely the time and size scales of interest for the latest generation of high-performance microelectronics. Another important phenomenon directly related to the small size of present devices is the existence of hot electrons whose dynamics is crucial to device performance. Fluid models are therefore adequate for large, slow components, but become almost useless for state-of-the-art designs now under development.

The most general approach to semiconductor modeling utilizes a microscopic, first principles description of charge carrier transport. The electron and hole kinetics are simulated by a Monte Carlo method that takes account of all major physical processes, including polar optical and acoustic scattering, interband transitions, and intraband scattering among satellite valleys for the upper energy bands. Scattering by plasmons and ionized impurities in the lattice are also important and must be included in certain cases. The charge distribution determined from the Monte Carlo transport calculation is coupled to a self-consistent Poisson solution for the electric fields in the device. This approach has been successfully applied to the simulation of multiband field-effect transistors.

The main limitations in the coupled Monte Carlo/Poisson models have not been in the transport physics, but in the inability to accurately model realistic geometries, material interfaces, material dispersive properties, and transient electromagnetic effects. Recent 
advances in plasma simulation techniques are now making it possible to overcome these limitations. Within the past few years, initial efforts have been made to model semiconductors using a fully electromagnetic solution of Maxwell's equations, in place of the electrostatic approximation (Poisson's equation). Electromagnetic solutions have also been coupled with Monte Carlo transport for modeling subpicosecond microelectronic switches. Other investigations have addressed electromagnetic crosstalk, ultra-high-frequency dispersive effects, nonlinear space-charge effects and other plasma physics phenomena in semiconductor components. The considerable successes achieved in these studies indicate that a general physics capability for self-consistent simulation of microelectronics is now within reach.

The goals of this project have been two-fold: To incorporate new physics into a firstprinciples Monte Carlo device simulator, and to develop the simulator into a state-of-the-art numerical tool with open architecture and design for maximum flexibility; practicality on workstation/cluster environment, and complete portability among platforms.

\section{Importance to LANL's Science and Technology Base and National R\&D Needs}

Truly predictive device design is fundamental to the future success of the microelectronics industry. After discussions with a number of microelectronics companies, a recurring theme is the desire for more reliance on modeling and simulation to reduce the cost and time to bring a new product to market. The driving force behind the increased emphasis on TCAD (Technology Computer Aided Design) appears to be associated with "shrink technology" coupled with the increased complexity of fabrication.

In regard to the LANL mission, this Laboratory has always played a significant role in modeling of complex physical processes because of the depth of our capabilities. This project allows us to maintain and expand the technology base, while at the same time making a contribution to the competitive stance of U.S. industry. The importance of continued leadership and self-reliance in microelectronics, for both economic and national security reasons, needs no elaboration. This effort is consistent with current DOE/LANL missions, and also offers the opportunity to participate at the forefront of a crucial, highly visible, and rapidly growing scientific field. 


\section{Scientific Approach and Results}

\section{A. Comparison of Device Simulators}

We have compared the models in, and simulation results from, the bulk codes MOCABULK and SLAPSHOT. Bulk codes provide an excellent starting point for comparison since they are simpler than the device codes for which they are the basis. These particular codes are representative of full-band and analytic-band codes.

SLAPSHOT uses a sophisticated analytic fit to the band structure and density of states in its discrete event simulation of charge carriers in bulk silicon. The analytic band and constant gamma technique permits the analytic integration of the equations of motion between free flight times; the analytic band and density of states permits an efficient $\mathrm{k}$-dependent selection of state after a collision. The collisional phonons are categorized by their dispersion branch and its sophisticated ionized impurity scattering model uses Brooks-Herring, ConwellWeisskopf, and semi-empirical degeneracy models. SLAPSHOT's field- and dopingdependent mobilities are in excellent agreement with experimental data.

MOCABULK uses a full band and density of states in its discrete time-step simulation of charge carriers in bulk silicon. The full-band and modified-constant time technique permits the discrete integration of the equations of motion; a band structure table sorted on energy permits efficient energy-dependent selection of state after a collision. Its collision phonons are categorized by scattering process and its ionized impurity scattering model uses Ridley's statistical screening model. MOCABULK's field- and doping-dependent mobilities are in fair agreement with experimental data.

Both codes represent an interesting collection of compromises between fidelity and efficiency. Design decisions made in each code inexorably limit other design choices, and ultimately force the codes to take the shape they have. This unfortunately makes the job of evaluating and perhaps mixing models from these codes hard, and challenges us to find a way to factor out the essential, loosely coupled parts of the model.

The comparison of physics models and solutions techniques used in different codes is an important exercise to understand their effects in device simulation. It forms the first step toward the development of the standard platform for Monte Carlo device simulators which will be instrumental to the development and benchmarking of the hierarchy among device simulators. 


\section{B. Device Simulator Using Object Oriented Programming Approach}

The object-oriented device code uses models based on the models in SLAPSHOT and MOCABULK. Currently, the code solves the Boltzmann transport equation using an ensemble particle model, with a modified constant time technique for finding collision times a la MOCABULK. The general field class is used to implement both full and analytic fit bands as in MOCABULK and SLAPSHOT, respectively. Coding of the scattering and final selection models is underway, based on the energy-dependent models used in MOCABULK. Momentum-dependent models, perhaps based on SLAPSHOT, can be implemented later. Completion of the scattering and final state models will give a working bulk code. The modular nature of the code provides a natural development path from bulk to device codes via the addition of objects which implement boundary conditions and field solvers.

\section{Physics of Silicon-Germanium Compounds for Microelectronic Devices}

The scaling of silicon MOSFET channel lengths to increase speed and drive current has pushed the limit of gate length towards $0.1 \mathrm{~mm}$. An alternative approach to enhance drive current is by increasing the mobility of the charge in the channel. Recent reports have shown that electron and hole mobilities are enhanced when they flow in strained $\mathrm{Si} / \mathrm{SiGe}$ channel. The mobility is enhanced due to strain induced change in band structure of the material systems. In our previous work, we simulated electron transport in strained Si on a relaxed SiGe system. Monte Carlo simulations have been performed for both bulk and device cases and the electron mobility is found to be improved by a factor of 1.8 as compared to electron mobility in the $\mathrm{Si}$ inversion layer for the same effective field. Our simulation results show good agreement with recent experiments. Using SLAPSHOT, we extended our work to optimize the strained $\mathrm{Si} / \mathrm{SiGe}$ MOSFET for best performance. In the second phase of our work, we developed a self-consistent Schrodinger and Poisson simulator to study hole transport in $\mathrm{Si}$ and strained SiGe material systems.

The quantum mechanical nature of a two-dimensional (2D) gas in the quantum well formed between $\mathrm{Si}$ and $\mathrm{SiGe}$ heterostructure can not be addressed by classical calculations. The sub-band energies are calculated using effective mass approximations. The effective masses of the strained SiGe alloy has been computed using the $\mathrm{k} \cdot \mathrm{p}$ perturbation method. Although the effective mass approximation is not quite valid for hole bandstructure, the fullband formalism is still a formidable problem for realistic computation. The carrier concentrations are calculated from sub-band dispersion knowing the doping concentrations and the one-dimensional Poisson equation is then solved using the successive over-relaxation 
method. The iteration between Schrodinger and Poisson equations is continued until a convergence is achieved. The quantum-mechanical solution is computed at small intervals of gate bias to obtain the capacitance versus voltage characteristics for the entire range of accumulation-to-inversion region. A significant difference has been found between quantum mechanical and classical quasi-static capacitance when the doping concentration is high and oxide thickness is low ( typical case for a deep submicron device). Due to the quantum mechanical nature of hole gas, the effective oxide thickness also increases, which results in an inferior capacitance. Effective hole mobility has been calculated using the 2D scattering rate that includes phonon (acoustic and optical), surface roughness, and alloy scattering processes. Good agreement has been obtained for inversion-layer, effective hole mobility as a function of effective field. The mobility in strained $\mathrm{SiGe}$ alloy increases due to strain-induced reduction of interband scattering and reduced effective masses, though alloy scattering represents some degradation of the mobility.

Initial results show significant enhancement of hole mobility in SiGe system over conventional Si pMOSFET. The quantum-mechanical capacitance has been calculated for different strain conditions. The effective hole mobility for strained SiGe pMOSFET increases with Ge mole fraction. However, the upper limit of Ge mole fraction is constrained by critical limit thickness, which is typically $100 \mathrm{~A}$ for a mole fraction of 0.4 . Using the quantummechanical simulator the optimum structure for best performance has been analysed. More experimental investigation is required in order to validate the models in our simulator.

\section{Publications}

1. Rashed, Mahbub; Jallipalli, S.; Shih, W.; Kwan, Thomas; and Maziar, Christine, "Monte Carlo Simulation of Electron Transport in Strained and Unstrained SiGe on Si Substrate," Journal of Applied Physics, submitted (1995).

2. Rashed, Mahbub; Jallepalli, S.; Shih, W.; Kwan, Thomas; and Maziar, Christine, "Monte Carlo Simulation of Deep Submicron MOSFETs with Carrier-Carrier Scattering and a New Ionized Impurity Scattering Model, Journal of Applied Physics, submitted (1995). 